\title{
A41 SELECTIVE INVOLVEMENT OF ERK AND JNK MAP KINASES IN THE SYNOVIAL TISSUE OF PATIENTS WITH EARLY ARTHRITIS
}

D de Launay, M G H van de Sande, G P M van de Sande, C A Wijbrandts, P P Tak, K A Reedquist Division of Clinical Immunology and Rheumatology, Academic Medical Center, University of Amsterdam, The Netherlands

10.1136/ard.2010.129593c

Background Little is known about the contribution of mitogen-activated protein kinase (MAPK) to disease in rheumatoid arthritis (RA), although inhibitors targeting one of these enzymes (p38) have already entered the clinic. We have investigated synovial MAPK activation status in disease-modifying antirheumatic drug (DMARD)-naïve patients with early arthritis.

Methods 50 patients with DMARD-naïve early arthritis (disease duration $<1$ year) were prospectively followed and diagnosed at baseline and after 2 years according to criteria for undifferentiated arthritis (UA), RA or spondyloarthritis (SpA). Synovial biopsies from actively inflamed joints were obtained at baseline by needle arthroscopy and examined by immunohistochemistry for expression and phosphorylation of p38, extracellular signal-regulated kinase 1 and 2 (ERK 1/2) and c-Jun-N-terminal kinase/mitogen-activated protein kinases (JNK/MAPKs) using computer-assisted image analysis. Results were compared between patients with different diagnoses and disease outcomes.

Results Activation of ERK was enhanced at inclusion in patients meeting RA criteria after 2 years compared with SpA $(p<0.05)$ and UA $(n=6)(p<0.005)$. JNK activation was significantly higher in RA than in SpA $(p<0.005)$ and UA $(p<0.01)$. p38 activation was similar between diagnostic groups. Logistic regression analysis demonstrated that synovial JNK activation, but not $\mathrm{p} 38$ or ERK activation, predicts fulfilment of RA classification criteria after 2 years $\left(R^{2}=0.59, p=0.02\right)$. Comparing patients diagnosed with UA at baseline who fulfilled RA classification criteria after 2 years with those who remained UA, activation of JNK ( $p<0.005)$ but not $\mathrm{p} 38$ or ERK was significantly enhanced. Activation of ERK $(p<0.01)$ and JNK $(p<0.01)$ at baseline was also enhanced in RA patients with progressive joint destruction as assessed by comparison of x-rays at baseline and after 2 years. Comparing all patients with early arthritis, activation of p38 ( $<<0.05)$, ERK $(p<0.005)$ and JNK $(p<0.001)$ was elevated in patients with erosive disease.

Conclusions In patients with early arthritis, elevated ERK and JNK activity distinguish RA from other forms of arthritis and JNK activation is already elevated in patients with RA, even before classification criteria of RA are met. Activation of MAPKs is also associated with the development of erosive disease and JNK activation predicts the development of erosive disease in early arthritis patients. Together, our data suggest that strategies targeting ERK and JNK, rather than p38, may be beneficial in treating RA early in the disease process. 